\title{
Review Article \\ The Emerging and Diverse Roles of Src-Like Adaptor Proteins in Health and Disease
}

\author{
Nikolett Marton, ${ }^{1}$ Eszter Baricza, ${ }^{1}$ Barbara Érsek, ${ }^{1,2}$ Edit I. Buzás, ${ }^{1}$ and György Nagy ${ }^{1,3}$ \\ ${ }^{1}$ Department of Genetics, Cell- and Immunobiology, Semmelweis University, 4 Nagyvárad Square, Budapest 1089, Hungary \\ ${ }^{2}$ MTA-SE "Lendület" Experimental and Translational Immunomics Research Group, 4 Nagyvárad Square, Budapest 1089, Hungary \\ ${ }^{3}$ Department of Rheumatology, Faculty of Medicine, Semmelweis University, 4 Frankel Leó Street, Budapest 1023, Hungary
}

Correspondence should be addressed to György Nagy; gyorgyngy@gmail.com

Received 28 September 2014; Revised 2 January 2015; Accepted 5 January 2015

Academic Editor: Fulvio D’Acquisto

Copyright (C) 2015 Nikolett Marton et al. This is an open access article distributed under the Creative Commons Attribution License, which permits unrestricted use, distribution, and reproduction in any medium, provided the original work is properly cited.

\begin{abstract}
Although Src-like adaptor proteins (SLAP-1 and SLAP-2) were mainly studied in lymphocytes, where they act as negative regulators and provide fine control of receptor signaling, recently, several other functions of these proteins were discovered. In addition to the well-characterized immunoregulatory functions, SLAP proteins appear to have an essential role in the pathogenesis of type I hypersensitivity, osteoporosis, and numerous malignant diseases. Both adaptor proteins are expressed in a wide variety of tissues, where they have mostly inhibitory effects on multiple intracellular signaling pathways. In this review, we summarize the diverse effects of SLAP proteins.
\end{abstract}

\section{Introduction}

Signal transducing adaptor proteins are a group of intracellular and transmembrane molecules which are crucial supplementary factors of signaling pathways. They mediate interactions between different molecules and contribute to the formation of signaling complexes. Adaptor proteins lack enzymatic activity and interaction domains enable them to connect with other molecules (e.g., proteins, lipids). Srclike adaptor protein 1 (SLAP-1) was cloned in a yeast twohybrid screen with the cytoplasmic domain of the receptor tyrosine kinase ephrin type-A receptor 2 as decoy $[1,2]$. SLAP proteins are not identical in size; SLAP-1 (or often quoted as SLAP) contains 276 amino acids while SLAP-2 consists of 261 amino acids (Figure 1). SLAP-1 and SLAP-2 contain common $\mathrm{SH} 2-\mathrm{SH} 3$ domains. $\mathrm{SH} 2$ domains allow proteins to dock to phosphorylated tyrosine containing epitopes. SH3 domains bind to hydrophobic amino acid and proline rich molecules. SH domains of SLAP-2 form continuous $\beta$-sheet that stretches the modular domains [3].

SLAP proteins were named after Src family kinases which have the same SH sequences. Src protein tyrosine kinases (PTKs) are protooncogenes that play a role in cell proliferation, survival, and morphology. Unlike Src PTKs, SLAP molecules do not have tyrosine kinase domains. In addition to the myristoylated amino-terminal and $\mathrm{SH}$ domains, SLAP family members contain unique carboxyterminal sequences as well. SLAP-1 has a longer carboxytail than SLAP-2, but the N-terminal is longer in SLAP-2. Myristoylated part of the SLAP promotes the association with membranes, while isoforms without the myristoylated Nterminal are located in the nucleus (Figure 1). Human SLAP is coded by a $64 \mathrm{~kb}$ intron of the thyroglobulin gene, on chromosome 8q24.23 in the candidate territory for a recessive demyelinating neuropathy. Sequence analysis could not find any mutations suggesting that this gene is not responsible for the disease [4]. Human SLA2 gene is located on 20q11.23 [1].

Human SLAP-1 and SLAP-2 molecules show sequence homology similarly to mouse SLAP proteins. SH domains are the most similar parts of the two SLAP molecules, in which the sequence homology is $59 \%$, while the $\mathrm{N}$-terminals are the most different parts; they have only $19 \%$ identity. SLAP-2 has a shorter alternative splice variant, called SLAP$2 \mathrm{v}$. This isoform contains only 210 amino acids due to the deletion of $50 \mathrm{bp}$ from exon 6, which results an alternative reading frame. The splicing variant molecule does not have 


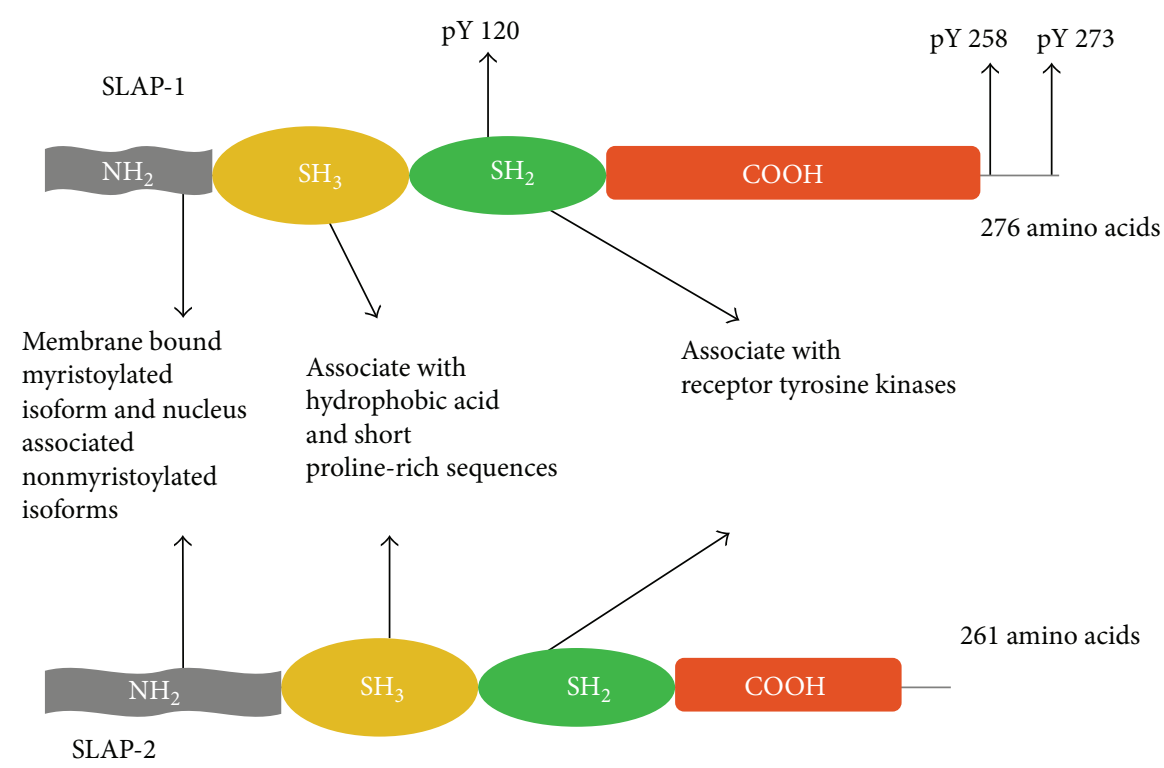

Figure 1: The schematic structure of SLAP molecules. SLAP-1 and SLAP-2 contain unique carboxy-terminal sequences, common SH2SH3 domains and amino-terminals which exist in myristoylated and nonmyristoylated isoforms. SH2 domains help molecules to bind to phosphorylated tyrosine containing epitopes. The SH3 domains connect the proline and hydrophobic amino acid containing molecules.

TABLE 1: The expression of SLAP proteins in different tissues and cell lines.

\begin{tabular}{|c|c|c|c|c|c|}
\hline Tissue/organ & Expressed molecule & Reference & Cell line & Expressed molecule & Reference \\
\hline Blood & SLAP-1, SLAP-2 & {$[1,2]$} & $\mathrm{BaF} 3$ & SLAP-1, SLAP-2 & {$[5]$} \\
\hline Bone marrow & SLAP-1, SLAP-2 & {$[9,10]$} & BJAB & SLAP-2 & {$[35]$} \\
\hline Brain & SLAP-1 & {$[9]$} & D011.10T & SLAP-2 & {$[5]$} \\
\hline Colon & SLAP-2 & {$[10]$} & EL4 & SLAP-1 & {$[9]$} \\
\hline Heart & SLAP-1, SLAP-2 & {$[10]$} & FLI-1 transformed erythroblasts & SLAP-1 & {$[8]$} \\
\hline Kidney & SLAP-1 & {$[9]$} & $\mathrm{HeLa}$ & SLAP-1 & {$[7]$} \\
\hline Liver & SLAP-1 & [9] & HL60 & SLAP-1 & {$[12]$} \\
\hline Lung & SLAP-1, SLAP-2 & {$[9]$} & Jurkat & SLAP-1, SLAP-2 & {$[7,35]$} \\
\hline Lymph nodes & SLAP-1 & {$[7]$} & NB-4 & SLAP-1 & {$[12]$} \\
\hline Pancreas & SLAP-1 & {$[10]$} & NIH3T3 & SLAP-1 & {$[10]$} \\
\hline Placenta & SLAP-1, SLAP-2 & {$[10]$} & RBL-2H3 & SLAP-1 & {$[11]$} \\
\hline Prostate & SLAP-2 & {$[10]$} & U.937 & SLAP-1 & {$[12]$} \\
\hline Skeletal muscle & SLAP-1, SLAP-2 & {$[9,10]$} & & & \\
\hline Small intestine & SLAP-1, SLAP-2 & {$[10]$} & & & \\
\hline Skin & SLAP-1, SLAP-2 & {$[10]$} & & & \\
\hline Spleen & SLAP-1, SLAP-2 & {$[9,10]$} & & & \\
\hline Thymus & SLAP-1, SLAP-2 & {$[29,30]$} & & & \\
\hline
\end{tabular}

any c-Cbl interacting site. The biological relevance of SLAP$2 \mathrm{v}$ is yet unknown $[5,6]$. Although expression of SLAP-1 and SLAP-2 mRNAs has been most extensively studied in lymphocytes, they are also expressed by numerous human and murine tissues and cell lines [1, 2, 7-12] (Table 1). Several proteins have been reported to interact with SLAP-1 and SLAP-2 [5, 8, 9, 13-15] (Figure 2). SLAPs are involved in a broad range of cellular processes, for example, lymphocyte development, neuronal excitotoxicity and platelet activation. SLAP molecules may participate in several pathological conditions of the immune system as well. In the present review, we will discuss the role of both SLAP proteins in different cell types and overview our current understanding regarding their relevance in pathological conditions.

\section{SLAP Proteins in Immune Cells}

2.1. SLAP-1 in T Lymphocytes. SLAP proteins were extensively investigated in lymphocytes where they are strongly expressed. SLAP-1 is involved in the regulation of the TCR signal transduction pathway. TCR consists of a ligand binding $\alpha \beta$ heterodimer and the CD3 complex that includes the $\gamma \varepsilon$ 


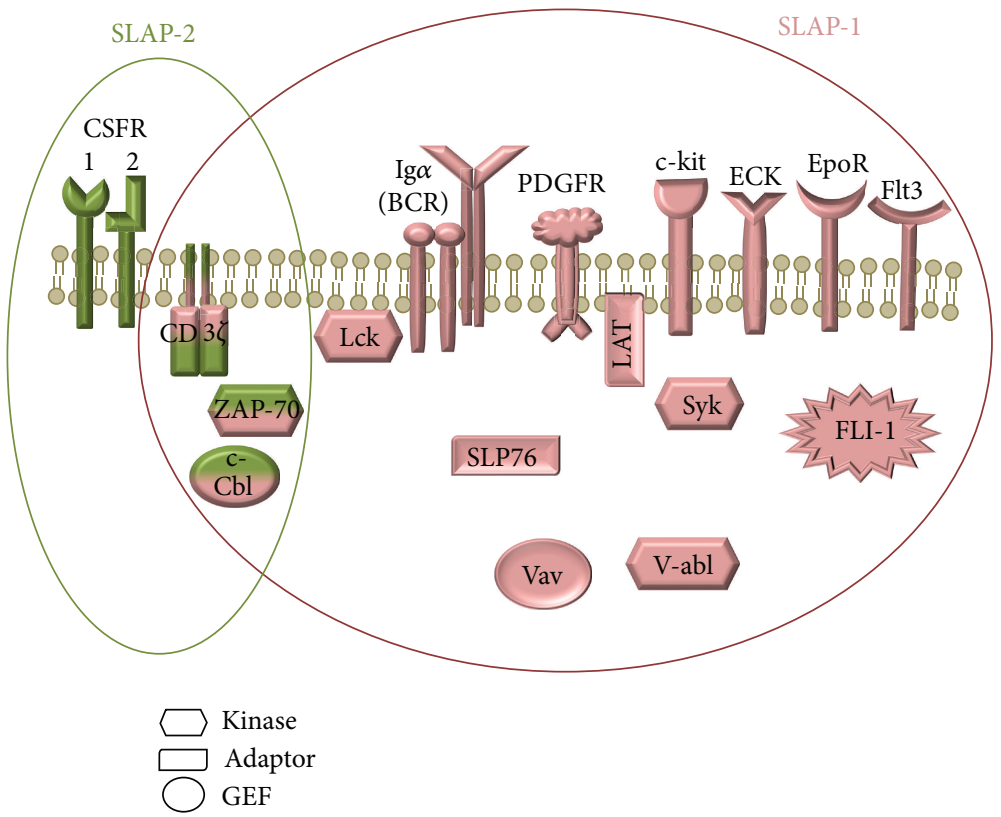

FIGURE 2: The interaction partners of SLAP-1 (pink) and SLAP-2 (green). Several proteins have been reported to interact with SLAP-1 molecule: c-Cbl, CD3 $\zeta$ chain, ECK, EpoR, Ig $\alpha$, LAT, Lck, PDGFR, SLP-76, Syk, v-abl, Vav (protooncogene vav), ZAP70, c-kit, Flt3, and FLI. SLAP-2 interacts with c-Cbl, $\mathrm{CD} 3 \zeta$ chain, CSFR, and ZAP70.

and $\delta \varepsilon$ heterodimers and the $\zeta \zeta$ homodimer. The expression of the $\zeta$-chains is about the $10 \%$ of the other subunits of the TCR complex. SLAP-1 may interact with many molecules involved in the TCR signal transduction (ZAP-70, Syk, LAT, $\mathrm{CD} 3 \zeta$-chain, Vav, and Lck) through the $\mathrm{SH} 2$ domain $[9,16]$.

SLAP-1 reduces the production of IL-2 and the transcription of NFATcl and AP-1, thereafter functions as a negative regulator of the TCR signaling; both $\mathrm{SH} 2$ and $\mathrm{SH} 3$ domains are necessary for this effect. However, upon ionomycin or PMA activation of lymphocytes, these inhibitory effects are absent suggesting that SLAP-1 regulates the proximal part of the TCR pathway [7]. SLAP may associate with the $\mathrm{N}$-terminal of the E3 ubiquitin ligase $\mathrm{c}$ - $\mathrm{Cbl}$ in a tyrosine phosphorylation independent way [16]. The simultaneous expression of SLAP- 1 and $\mathrm{c}-\mathrm{Cbl}$ promotes the ubiquitination and degradation of the $\zeta$-chain, consequently enhancing the recycling and preventing the accumulation of the receptor complexes [17]. By contrast, similarly to SLAP -/- lymphocytes, c-Cbl -/- lymphocytes overexpress the $\zeta$-chain due to its abolished degradation [18]. For successful operation, SLAP-1 requires the phosphorylation of the cytoplasmic domains of the TCR $\zeta$-chains and the activation of Lck, but not of ZAP70 [19]. It has been shown that the phosphorylated proportion of the $\zeta$-chains is slightly traceable in Lck -/lymphocytes [20,21]. SLAP colocalizes with early endosomes according to confocal microscopy images [7] (Figure 3). It is noteworthy that downregulation of $\zeta$-chain of T-cells has been observed in many pathological conditions including rheumatoid arthritis (RA), systemic lupus erythematosus (SLE), human immunodeficiency virus (HIV) infection, and various cancers [22-28].

Thymic selection is essential in shaping the peripheral $\mathrm{T}$ cell repertoire. The central role of SLAP in lymphocyte development has been also described. The fate of the early lymphocytes depends on the TCR-mediated signals: both too strong and too week signals through the TCR lead to cell death (during positive and negative selections, resp.). The expression of SLAP is strictly regulated during the thymic development of lymphocytes. It is expressed at low levels in CD4- CD8- cells and at high levels in CD4+ CD8+ thymocytes. SLAP as a regulator of the TCR expression plays a pivotal role in the downregulation of TCR complexes of the developing lymphocytes. Enhanced positive selection was observed in SLAP deficient mice. Moreover, the apoptosis of ZAP70 -/ - cells was inhibited in the absence of SLAP [29]. It has been reported that the proline rich-sequence (PRS) of CD3e act together with SLAP in the regulation of TCR expression in CD4+ CD8+ thymocytes. CD $3 \varepsilon$ PRS deficient cells were unable to degrade the $\zeta$-chain [30]. In double positive thymocytes, the chains of the TCR complex are constitutively ubiquitinated, but the ubiquitination is absent in mature cells. It has been shown that CD3e PRS, Lck, c-Cbl, and SLAP are required for the ubiquitination and degradation of the $\zeta$-chains. In the absence of ubiquitination, both the lysosomal sequestration and degradation are failed, and TCR chains are upregulated in CD4+ CD8+ lymphocytes. In addition, modified TCR complex ubiquitination influences the formation of the immunological synapse and alters the selection of the immature cells [31]. The lack of SLAP increases the avidity of the TCR which leads to the negative selection of antigen specific CD8+ cells. All these data suggest that SLAP plays a major role in the TCR repertoire configuration [32].

2.2. SLAP-1 in B Lymphocytes. SLAP is associated with c-Cbl in B lymphocytes, leading to BCR recycling [33] (Figure 4). 


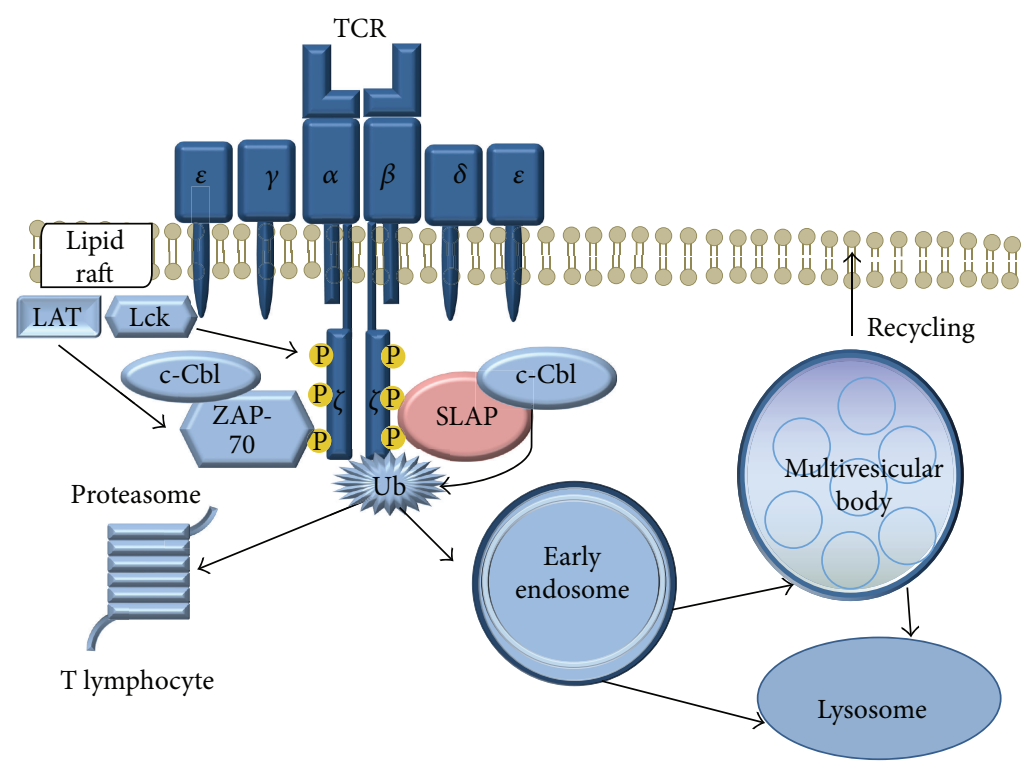

Figure 3: The role of SLAP in T lymphocytes.

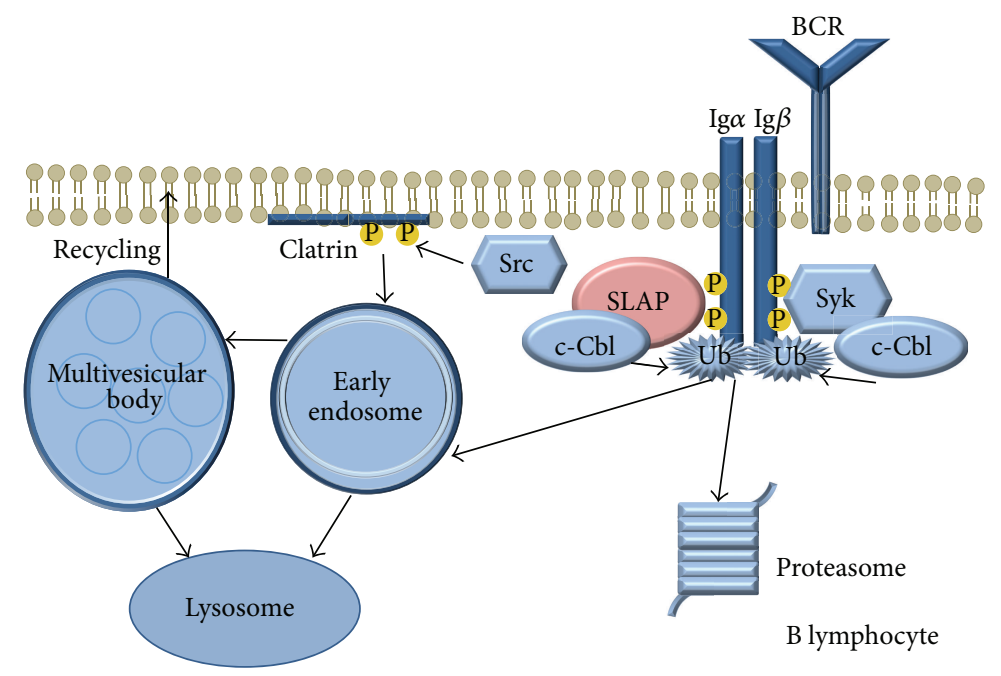

FIgURE 4: The role of SLAP in B lymphocytes.

SLAP deficiency increases the BCR levels of immature B cells in HEL-specific MD4- transgenic mice, which is a frequently used model to study naïve B lymphocytes. In this model the upregulated receptor complex levels lead to increased signal transduction. SLAP deficient mice have an increased number of splenic B cells, but the surface expression of BCR and IgM of mature lymphocytes is decreased. Furthermore, the activation induced calcium flux is diminished in SLAP KO B cells. In virtue of the previous data SLAP regulates the level of BCR $s$ which is essential for the adequate development and function of B cells [34].

2.3. SLAP-2 in $T$ and B Lymphocytes. Similarly to SLAP1, SLAP-2 also has an effect on lymphocyte receptor signalization. SLAP-2 has a negative regulatory role on the antigen receptor signaling of $\mathrm{T}$ and $\mathrm{B}$ lymphocytes. The overexpression of SLAP-2 reduces the surface levels of CD3 [5]. The overexpression of SLAP-1 and SLAP-2 inhibits the upregulation of CD69 after antigen receptor cross-linking. CD69 is an inducible cell surface glycoprotein, upregulated during lymphocyte activation. SLAP-2 suppresses the antigen binding induced calcium influx in $\mathrm{T}$ (Jurkat) and $\mathrm{B}$ (BJAB) cell lines. SLAP-2 reduces the CD69 expression in Jurkat cells (61\%) more significantly than in BJAB cells $(28 \%)$. Similarly to SLAP-1, SLAP-2 does not impair the ionomycin and PMA induced signalization [35]. SLAP-2 is associated with ubiquitin ligase c-Cbl similarly to SLAP-1, deletion of the carboxy-terminal of SLAP-2 was reported to inhibit this connection [35]. In activated Jurkat cells SLAP-2 binds to cCbl, ZAP-70, and CD3 $\zeta$ in a phosphorylation independent manner [1]. The coexpression of SLAP-2 and ZAP-70 or Syk in T-cell lines lead to the degradation of both kinases. Thus, 


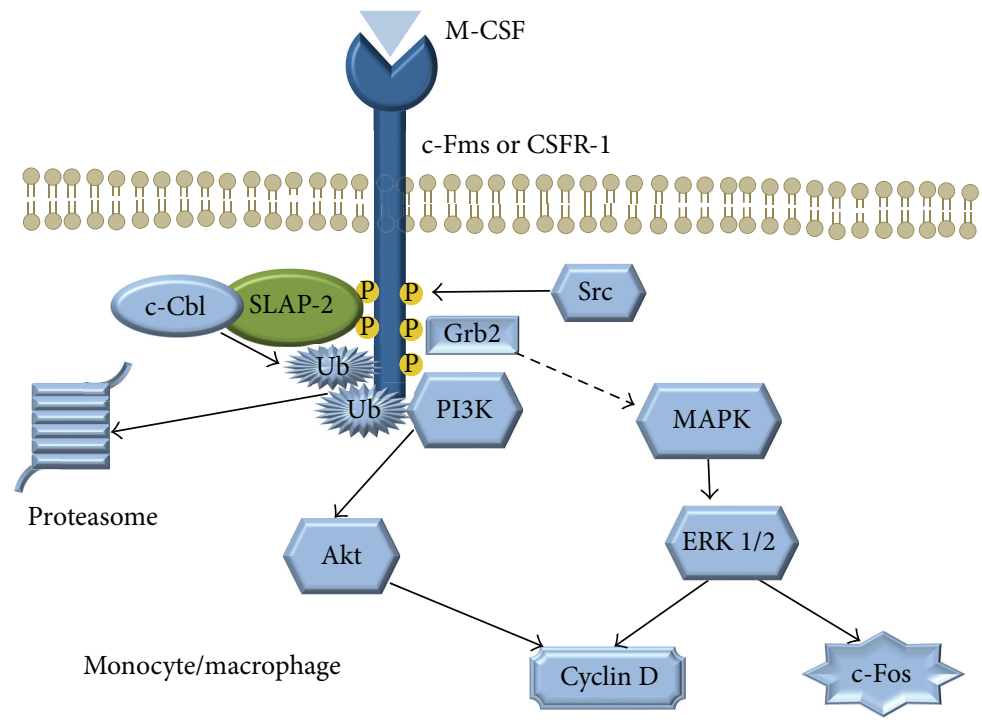

FIgURE 5: The role of SLAP-2 in monocytes/macrophages.

SLAP-2 induces the c-Cbl dependent degradation of tyrosine kinases and downregulates CD3 expression.

2.4. SLAP Molecules in Monocytes and Dendritic Cells. SLAP2 is expressed in human monocytes and bone marrow cells $[1,35]$, but not in CSF1 independent monocyte cell lines such as RAW264.7 [5]. It was shown that SLAP-2 may bind to both c-Fms and c-Cbl, and several domains of SLAP-2 are involved in this interaction (Figure 5). The overexpression of SLAP-2 in murine bone marrow reduced the M-CSF-induced tyrosine phosphorylation [36]. SLAP-2 downregulates the cFms signaling through a c-Cbl dependent internalization and degradation of the CSF-1R, providing a negative feedback of the M-CSF pathway [37]. In addition, c-Fms stimulation induces the phosphorylation of the serine rich N-terminal domain of the SLAP-2 by a JNK dependent pathway [36].

The activation-induced GM-CSFR downregulation is attenuated in SLAP-1 and SLAP-2 deficient bone marrow dendritic cells (BM-DC), which is associated with enhanced MAP/Erk and Akt pathways upon GM-CSF stimulation. The inhibited activation through GM-CSFR impairs the bone marrow derived dendritic cell maturation. SLAP-1 and SLAP-2 deficient BM-DC cells produce less IL-12 and TNF$\alpha$ upon lipopolysaccharide (LPS) stimulation and induce significantly less IFN- $\gamma$ secretion of T-lymphocytes than the wild type cells [38]. These data suggest that SLAP proteins are necessary for monocyte and dendritic cell maturation and activation.

2.5. SLAP Proteins in Mast Cells. SLAP has a prominent role in the regulation of intracellular signal transduction in mast cells. SLAP-specific small interfering RNAs inhibit the effect of dexamethasone on the phosphorylation of PLC $\gamma$, LAT, Syk, and ERK [39]. Actinomycin D inhibits the transcription of SLAP following dexamethason treatment, suggesting that the upregulation for SLAP-1 upon glucocorticoid treatment occurs at the transcriptional level. Glucocorticoid receptor activation is required for the elevation of the SLAP transcription [40]. SLAP-1 plays a role in the negative regulation of antigen-stimulated mast cells as well. Upon antigen stimulation of the RBL-2H3 mast cell line, elevated transcription of the SLA gene and upregulation of the SLAP-1 (but not SLAP-2) protein were reported. An increased amount of SLAP-1 was detected after 60 minutes stimulation and it reached the maximum after 2-3 hours. By contrast, after silencing of SLAP, increased IL-3 and MCP-1 production was detected. Knock-down of SLAP by using siRNA increases the expression of FceRI [39]. Thus, SLAP appears to be a crucial regulator of mast cell function.

\section{The Function of SLAP Molecules in Other Cell Types}

3.1. SLAP-1 in Osteoclasts. Osteoclasts play a fundamental role in the pathogenesis of osteoporosis. Inhibiting the development and activation of osteoclasts is currently the gold standard therapeutic strategy in this disease. Although today several antiosteoporotic drugs are widely used in the clinical practice, the effect of these medications on increasing bone density and strength is moderate. There is an inverse correlation between both SLAP-1 and SLAP- 2 and the tartrate-resistant acid phosphatase (osteoclastogenesis marker) mRNA expression. The level of osteoclast-specific protein mRNAs (e.g., catepsin K and MMP-9) are elevated in the SLAP-1 -/- preosteoclasts. SLAP-1 has an inhibitory effect on the MAP kinase pathway which is initiated with the binding of M-CSF to its receptor c-Fms. This receptor is a tyrosine kinase transmembrane protein which accumulates in lipid rafts where it associates with SLAP-1. The lack of SLAP enhances osteoclastogenesis without changing the resorptive function of the individual cells [41]. In addition, SLAP-1 deficiency increases the apoptosis of the mature 
polykariotic osteoclasts without altering the viability of the precursors. According to our current understanding, SLAP-1 has a regulatory effect on osteoclastogenesis and mature cell survival through the M-CSF pathway [41].

3.2. SLAP Molecules in Platelets and Fibroblasts. SLAP-2 is expressed in human platelets and may associate with Syk, c$\mathrm{Cbl}$, and LAT. After its activation, SLAP-2 promotes Syk and c-Cbl to approach their substrates. SLAP-2 inhibits glycoprotein VI (GPVI) initiated signal transduction of platelets through the connection with $\mathrm{c}$-Cbl [42]. This inhibitory effect on thrombocyte activation is similar to those described previously in lymphocytes $[1,5]$.

SLAP may also associate with platelet derived growth factor receptor (PDGFR) in NIH3T3 mouse embryonic fibroblast cell line. Overexpression of SLAP in NIH3T3 cells inhibits PDGF-induced mitogenesis [43] suggesting that SLAP is a negative regulator of growth factor initiated signaling.

3.3. SLAP-1 in Neurons. Although in embryonic rat telencephalon sections SLAP-1 mRNA is absent in migrating neurons, it is highly and selectively expressed in neurons which have reached their final location. SLAP-1 has a characteristic expression pattern during the development of the cortex. It is expressed mostly by deeper stratum cells especially by pyramid cells. Pyramid cells are typically located in the deeper layers of the cortex, and they have connection with the subcortical areas. It is not yet clear whether SLAP1 plays a role in the axon guidance during development but apparently has a remarkable expression. There is also a possible interaction between SLAP-1 and EphA receptors [44].

Western blot analysis and immunolabeling of rat brain extract showed that SLAP-1 is located in the postsynaptic membrane. SLAP-1 has an association with EphAs and NMDARs have a connection with EphBs $[44,45]$. EphB may excite the enlistment of EphAs and promote the recruitment of SLAP-1 and NMDARs. Despite the analogous localization, SLAP-1 does not affect the baseline activity of NMDARs through a Src-dependent stimulation of the receptors. SLAP1 may have a role in the negative feedback which regulates the number of the NMDARs and prevent excitotoxicity. This effect of SLAP-1 is based on the proteasomal degradation of redundant NMDARs in neurons [46]. The described role of SLAP in neurons seems to be essential for the adequate neuronal functions.

\section{SLAP in Pathological Conditions}

4.1. The Role of SLAP in Rheumatoid Arthritis. Rheumatoid arthritis (RA) is a common autoimmune disease that is associated with progressive disability and systemic complications. Symmetric synovial inflammation of multiple joints, especially the small joints of the hands and the feet, is characteristic for the disease. The ongoing inflammation leads to cartilage destruction, bone erosions, and subsequent joint deformities. Although the current treatment strategy, especially the use of biologicals, improved largely the outcome of the disease, still only a small portion of the patients are in sustained remission and systemic complications (including cardiovascular risk) still represent a significant challenge. TNF- $\alpha$ plays an essential role in the pathogenesis of RA through promotion of angiogenesis, suppression of regulatory $\mathrm{T}$-cell activation, and cytokine and chemokine expression.

According to the recently published data of our research group, TNF- $\alpha$ treatment downregulates the expression of $\zeta$ chain of CD4 T-lymphocytes reversibly and selectively in a dose dependent way. Decreased $\zeta$-chain expression leads to the hyporesponsiveness of T-cells. TNF- $\alpha$ induces the expression of SLAP which promotes the proteasomal but not the lysosomal degradation of the $\zeta$-chain. Silencing SLAP with short interfering RNAs inhibits the TNF- $\alpha$ induced $\zeta$ chain degradation. TNF- $\alpha$ treatment does not alter the SLAP mRNA level, suggesting that TNF- $\alpha$ controls SLAP activity through miRNA mediated posttranscriptional silencing. CD4+ T-lymphocytes isolated from RA patients expressed more than 2-fold higher SLAP levels than the T-cells of healthy donors. TNF- $\alpha$ treatment enhances the expression of SLAP in the CD4+ T-lymphocytes of healthy donors and DMARD treated RA patients but does not alter the expression of CD4+ T-cells isolated from biological DMARD (etanercept, certolizumab pegol) treated patients [47].

A spontaneous mutation in ZAP70 protein uncoupled proximal TCR signal transduction and led to severe symmetrical arthritis in SKG mice upon exposure to zymosan. According to recently published data, SLAP deficiency dramatically reduced both the incidence and severity of zymosan-induced chronic autoimmune arthritis in SKG mice [48]. The protective role of SLAP deficiency was associated with the increased number of regulatory T-cells and decreased amount of Th17 cells [48].

4.2. The Role of SLAP in Malignant Diseases and in Erythropoiesis. SLAP expression is increased in several cancers including chronic myeloid leukemia (CML), chronic lymphocytic leukemia (CLL), and prostate cancer. By contrast, its expression is decreased in acute myeloid leukemia (AML), myeloma, and colon cancer [13]. Furthermore, SLAP is associated with several oncogenic signaling pathways.

It was recently reported that the transmembrane protooncogene c-kit is degraded through a SLAP dependent pathway. SLAP binds to the WT c-kit and initiates its ubiquitylation and degradation in the proteasome. By contrast, SLAP does not have a similar regulatory effect on the oncogenic c-kit variant (c-kit D816V). Oncogenic c-kit associates with SLAP and phosphorylates it on three different tyrosines (Y120, Y258, and Y273); the phosphorylated forms of SLAP do not alter the downstream signaling of the mutant c-Kit. These data suggest that SLAP regulates WT c-kit signaling, but the oncogenic variant escapes from this negative feedback regulation [49].

The Fms like tyrosine kinase (Flt3) is a receptor tyrosine kinase, which is predominantly expressed in hematopoietic progenitor cells. SLAP associates with both the WT and 
mutated, oncogenic Flt3 (Flt3-ITD). Silencing of SLAP with short interfering RNAs leads to an attenuated MAPK signal pathway. After ligand stimulation, SLAP colocalizes with the Flt3-ITD and targets it for c-Cbl dependent ubiquitylation and proteasomal degradation. About $30 \%$ of patients with AML have mutation in the Flt3 gene and gain of function mutations contribute to the initiation of AML. The expression of SLAP is increased in patients with acute promyelocytic leukemia (APL) carrying Flt3-ITD mutation as compared to the Flt3-WT [13].

FLI-1 is a transcription factor, a member of the E26 transformation-specific (ETS) protein family. Furthermore, the FLI-1 locus is an integration site for the Friend murine leukemia virus, which induces erythroleukaemia in responsive mice. SLAP protein and mRNA levels are overexpressed in FLI-1 transformed erythroblasts [8]. SLAP binds to both the phosphorylated and unphosphorylated forms of the erythropoietin receptor (EpoR). SLAP expression prevents the EPO-induced differentiation, whose effect is associated with the inhibition of STAT5 activation and BCL-X upregulation. Both STAT5 and BCL-X are critical in EPO-induced signaling [8]. SLAP may mediate the erythropoiesis in this manner.

According to recently published data, SLAP is expressed in colon epithelium, but it is significantly downregulated in colorectal tumors [15]. Interestingly, silencing of SLAP promotes tumor progression, while overexpression inhibits tumor growth and invasiveness. SLAP promotes the destabilization of the Src substrate ephrin type-A receptor 2 (EphA2) in the intestinal cells. EphA2 plays a critical role in the regulation of several intracellular signal pathways which mediate cell migration, invasion, and angiogenesis. The inhibitory effect of SLAP appears to be independent from $\mathrm{c}-\mathrm{Cbl}$ but has an association with the ubiquitination factor UBE4A and with the pTyr594-EphA2. These results suggest a tumor-suppressive effect of SLAP in colorectal cancers [15].

\section{Conclusions and Future Perspectives}

SLAP proteins are expressed in a variety of cell types which indicates a conserved function of these proteins. Both SLAP-1 and SLAP-2 have a prominent role in the negative regulation of several membrane bound receptors and receptor tyrosine kinases, thereafter SLAPs play a central role in the regulation of intracellular signal transduction and cell reactivity. The proper function of SLAPs is necessary for immunoreceptor repertoire configuration and helps to avoid uncontrolled cell activation, proliferation, and migration. SLAP molecules are involved in the ubiquitination of proteins, which may lead to proteasomal degradation. Selective regulation of SLAP molecules in different cell types may allow the fine control of cell activation and differentiation. Exploring the precise role of SLAP proteins will contribute to the understanding of many yet unknown physiological regulatory processes. In addition, tissue specific targeting of SLAP may provide valuable therapeutic approaches in diverse diseases including RA, osteoporosis, immunodeficiency, and different cancers.

\section{Abbreviations}

\begin{tabular}{|c|c|}
\hline AP-1: & Activator protein 1 \\
\hline AML: & Acute myeloid leukaemia \\
\hline BCLXL: & $\begin{array}{l}\text { B-cell lymphoma extra-large transmembrane } \\
\text { molecule }\end{array}$ \\
\hline BCR: & B cell receptor \\
\hline BJAB: & EBV-negative, Burkitt-like lymphoma \\
\hline c-Cbl: & $\begin{array}{l}\text { E3 Ubiquitin-protein ligase casitas B-linage } \\
\text { lymphoma protooncogene }\end{array}$ \\
\hline CD: & Cluster of differentiation \\
\hline CD3 $\zeta$-chain: & T-cell receptor complex zeta chain \\
\hline c-Fms: & Colony stimulating factor 1 receptor \\
\hline c-kit: & Mast/stem cell growth factor receptor \\
\hline CSF1: & Colony stimulating factor 1 \\
\hline DMARDs: & Disease modifying antirheumatic drugs \\
\hline ECK: & Ephrin type-A receptor 2 \\
\hline Eph: & Ephrin type receptor \\
\hline EphA2: & Ephrin type-A receptor 2 \\
\hline EphB: & Ephrin type-B receptor \\
\hline Epo: & Erythropoietin \\
\hline EpoR: & Erythropoietin receptor \\
\hline ERK: & Extracellular signal-related kinases \\
\hline FceRI: & Fc $\varepsilon$ receptor I \\
\hline FLI1: & $\begin{array}{l}\text { Friend leukemia integration } 1 \text { transcription } \\
\text { factor }\end{array}$ \\
\hline Flt3: & Fms-like tyrosine kinase 3 \\
\hline GM-CSFR: & $\begin{array}{l}\text { Granulocyte macrophage colony stimulating } \\
\text { factor receptor }\end{array}$ \\
\hline GPVI: & Glycoprotein VI \\
\hline HIV: & Human immunodeficiency virus \\
\hline $\operatorname{Ig} \alpha:$ & $\begin{array}{l}\text { B-cell receptor antigen complex-associated } \\
\text { protein alpha }\end{array}$ \\
\hline IFN $\gamma$ : & Interferon $\gamma$ \\
\hline IL: & Interleukin \\
\hline ITD: & Internal tandem duplications \\
\hline JNK: & c-Jun N-terminal kinase \\
\hline $\mathrm{kb}:$ & Kilobase \\
\hline LAT: & $\begin{array}{l}\text { Linker for activation of T-cells family } \\
\text { member }\end{array}$ \\
\hline Lck: & Protooncogene tyrosine-protein kinase \\
\hline MAP: & Microtubule associated protein \\
\hline MAPK: & Microtubule associated protein kinase \\
\hline MCP-1: & Monocyte chemotactic protein 1 \\
\hline M-CSF: & Macrophage colony stimulating factor \\
\hline MMP9: & Matrix metallopeptidase 9 \\
\hline mRNA: & Messenger ribonucleic acid \\
\hline miRNA: & Microribonucleic acids \\
\hline NFATc: & Nuclear factor of activated T-cells \\
\hline NMDAR: & N-methyl-D-aspartate receptor \\
\hline PDGF: & Platelet derived growth factor \\
\hline PDGFR: & Platelet derived growth factor receptor \\
\hline PLC $\gamma:$ & Phospholipase $\mathrm{C} \gamma$ \\
\hline PMA: & Phorbol myristate acetate \\
\hline PTK: & Protein tyrosine kinase \\
\hline RA: & Rheumatoid arthritis \\
\hline RNA: & Ribonucleic acid \\
\hline SH: & Src homology domain \\
\hline SLA: & Src-like adaptor \\
\hline
\end{tabular}


SLAP-1: Src-like adaptor protein 1

SLAP-2: Src-like adaptor protein 2

SLP-76: SH2 domain-containing protein of $76 \mathrm{kDa}$

Src: Sarcoma

STAT5: Signal transducer and activator of transcription 5

Syk: $\quad$ Spleen tyrosine kinase

Th17: $\quad$ Thelper 17

TNF- $\alpha$ : Tumor necrosis factor $\alpha$

TCR: T-cell receptor

UBE4A: Ubiquitin conjugation factor E4A

$\mathrm{V}$-abl: Tyrosine-protein kinase transforming protein Abl

Vav: Protooncogene vav

WT: Wild- type

ZAP70: Zeta-associated protein of $70 \mathrm{kDa}$.

\section{Conflict of Interests}

The authors declare no conflict of interests.

\section{Acknowledgment}

This work is funded by Grants OTKA NN111023 and NK84043.

\section{References}

[1] A. Pandey, N. Ibarrola, I. Kratchmarova et al., "A novel Src homology 2 domain-containing molecule, Src-like adapter protein-2 (SLAP-2), which negatively regulates $\mathrm{T}$ cell receptor signaling," The Journal of Biological Chemistry, vol. 277, no. 21, pp. 19131-19138, 2002.

[2] A. Pandey, H. Duan, and V. M. Dixit, "Characterization of a novel Src-like adapter protein that associates with the Eck receptor tyrosine kinase," The Journal of Biological Chemistry, vol. 270, no. 33, pp. 19201-19204, 1995.

[3] L. E. Wybenga-Groot and C. J. McGlade, "Crystal structure of Src-like adaptor protein 2 reveals close association of $\mathrm{SH} 3$ and $\mathrm{SH} 2$ domains through $\beta$-sheet formation," Cellular Signalling, vol. 25, no. 12, pp. 2702-2708, 2013.

[4] P. H. S. Meijerink, P. Yanakiev, I. Zorn et al., "The gene for the human Src-like adaptor protein (hSLAP) is located within the 64-kb intron of the thyroglobulin gene," European Journal of Biochemistry, vol. 254, no. 2, pp. 297-303, 1998.

[5] M. P. Loreto, D. M. Berry, and C. J. McGlade, "Functional cooperation between $\mathrm{c}-\mathrm{CbL}$ and Src-like adaptor protein 2 in the negative regulation of T-cell receptor signaling," Molecular \& Cellular Biology, vol. 22, no. 12, pp. 4241-4255, 2002.

[6] M. P. Loreto and C. J. McGlade, "Cloning and characterization of human Src-like adaptor protein 2 and a novel splice isoform, SLAP-2-v," Oncogene, vol. 22, no. 2, pp. 266-273, 2003.

[7] T. Sosinowski, A. Pandey, V. M. Dixit, and A. Weiss, "Src-like adaptor protein (SLAP) is a negative regulator of T cell receptor signaling," Journal of Experimental Medicine, vol. 191, no. 3, pp. 463-474, 2000.

[8] I. Lebigot, P. Gardellin, L. Lefebvre, H. Beug, J. Ghysdael, and C. T. Quang, "Up-regulation of SLAP in FLI-1-transformed erythroblasts interferes with EpoR signaling," Blood, vol. 102, no. 13, pp. 4555-4562, 2003.
[9] L. L. Dragone, L. A. Shaw, M. D. Myers, and A. Weiss, "SLAP, a regulator of immunoreceptor ubiquitination, signaling, and trafficking," Immunological Reviews, vol. 232, no. 1, pp. 218-228, 2009.

[10] L. E. Wybenga-Groot and C. J. McGlade, "RTK SLAP DOWN: the emerging role of Src-like adaptor protein as a key player in receptor tyrosine kinase signaling," Cellular Signalling, vol. 27, no. 2, pp. 267-274, 2015.

[11] T. Hiragun, Z. Peng, and M. A. Beaven, "Cutting edge: dexamethasone negatively regulates Syk in mast cells by up-regulating Src-like adaptor protein," The Journal of Immunology, vol. 177, no. 4, pp. 2047-2050, 2006.

[12] T. Ohtsuki, K. Hatake, M. Ikeda et al., "Expression of Src-like adapter protein mRNA is induced by all-trans retinoic acid," Biochemical and Biophysical Research Communications, vol. 230, no. 1, pp. 81-84, 1997.

[13] J. U. Kazi and L. Rönnstrand, "Src-like adaptor protein (SLAP) binds to the receptor tyrosine kinase Flt3 and modulates receptor stability and downstream signaling," PLOS ONE, vol. 7, no. 12, Article ID e53509, 2012.

[14] P. D. Kottaridis, R. E. Gale, M. E. Frew et al., "The presence of a FLT3 internal tandem duplication in patients with acute myeloid leukemia (AML) adds important prognostic information to cytogenetic risk group and response to the first cycle of chemotherapy: analysis of 854 patients from the United Kingdom Medical Research Council AML 10 and 12 trials," Blood, vol. 98, no. 6, pp. 1752-1759, 2001.

[15] C. Naudin, A. Sirvent, C. Leroy et al., "SLAP displays tumour suppressor functions in colorectal cancer via destabilization of the SRC substrate EPHA2," Nature Communications, vol. 5, article 3159, 2014.

[16] J. Tang, S. Sawasdikosol, J.-H. Chang, and S. J. Burakoff, “SLAP, a dimeric adapter protein, plays a functional role in T cell receptor signaling," Proceedings of the National Academy of Sciences of the United States of America, vol. 96, no. 17, pp. 9775-9780, 1999.

[17] M. D. Myers, L. L. Dragone, and A. Weiss, "Src-like adaptor protein down-regulates T cell receptor (TCR)-CD3 expression by targeting TCR $\zeta$ for degradation," Journal of Cell Biology, vol. 170, no. 2, pp. 285-294, 2005.

[18] M. Naramura, H. K. Kole, R.-J. Hu, and H. Gu, "Altered thymic positive selection and intracellular signals in Cbl- deficient mice," Proceedings of the National Academy of Sciences of the United States of America, vol. 95, no. 26, pp. 15547-15552, 1998.

[19] M. D. Myers, T. Sosinowski, L. L. Dragone et al., "Src-like adaptor protein regulates TCR expression on thymocytes by linking the ubiquitin ligase c-Cbl to the TCR complex," Nature Immunology, vol. 7, no. 1, pp. 57-66, 2006.

[20] T. Nakayama, A. Singer, E. D. Hsi, and L. E. Samelson, "Intrathymic signalling in immature $\mathrm{CD} 4^{+} \mathrm{CD} 8^{+}$thymocytes results in tyrosine phosphorylation of the T-cell receptor zeta chain," Nature, vol. 341, no. 6243, pp. 651-654, 1989.

[21] N. S. C. van Oers, N. Killeen, and A. Weiss, "ZAP-70 is constitutively associated with tyrosine-phosphorylated TCR $\zeta$ in murine thymocytes and lymph node T cells," Immunity, vol. 1, no. 8, pp. 675-685, 1994.

[22] M. Matsuda, A.-K. Ulfgren, R. Lenkei et al., "Decreased expression of signal-transducing CD3 zeta chains in T cells from the joints and peripheral blood of rheumatoid arthritis patients," Scandinavian Journal of Immunology, vol. 47, no. 3, pp. 254-262, 1998.

[23] L. Berg, J. Rönnelid, L. Klareskog, and A. Bucht, "Downregulation of the $\mathrm{T}$ cell receptor $\mathrm{CD} 3 \zeta$ chain in rheumatoid 
arthritis (RA) and its influence on T cell responsiveness," Clinical and Experimental Immunology, vol. 120, no. 1, pp. 174$182,2000$.

[24] S. N. Liossis, X. Z. Ding, G. J. Dennis, and G. C. Tsokos, "Altered pattern of TCR/CD3-mediated protein-tyrosyl phosphorylation in T cells from patients with systemic lupus erythematosus. Deficient expression of the T cell receptor zeta chain," The Journal of Clinical Investigation, vol. 101, no. 7, pp. 1448-1457, 1998.

[25] M. P. Nambiar, S. Krishnan, V. G. Warke, and G. C. Tsokos, "TCR zeta-chain abnormalities in human systemic lupus erythematosus," Methods in Molecular Medicine, vol. 102, pp. 4972, 2004.

[26] L. A. Trimble and J. Lieberman, "Circulating CD8 T lymphocytes in human immunodeficiency virus-infected individuals have impaired function and downmodulate $\mathrm{CD} 3 \zeta$, the signaling chain of the T-cell receptor complex," Blood, vol. 91, no. 2, pp. 585-594, 1998.

[27] L. A. Trimble, P. Shankar, M. Patterson, J. P. Daily, and J. Lieberman, "Human immunodeficiency virus-specific circulating CD8 T lymphocytes have down-modulated CD3zeta and CD28, key signaling molecules for T-cell activation," Journal of Virology, vol. 74, no. 16, pp. 7320-7330, 2000.

[28] C. Renner, S. Ohnesorge, G. Held et al., "T cells from patients with hodgkin's disease have a defective T-cell receptor $\zeta$ chain expression that is reversible by T-cell stimulation with CD3 and CD28," Blood, vol. 88, no. 1, pp. 236-241, 1996.

[29] T. Sosinowski, N. Killeen, and A. Weiss, "The Src-like adaptor protein downregulates the $\mathrm{T}$ cell receptor on $\mathrm{CD}^{+} \mathrm{CD} 8^{+}$ thymocytes and regulates positive selection," Immunity, vol. 15, no. 3, pp. 457-466, 2001.

[30] M. Mingueneau, A. Sansoni, C. Grégoire et al., "The prolinerich sequence of $\mathrm{CD} 3 \varepsilon$ controls $\mathrm{T}$ cell antigen receptor expression on and signaling potency in preselection $\mathrm{CD} 4^{+} \mathrm{CD} 8^{+}$ thymocytes," Nature Immunology, vol. 9, no. 5, pp. 522-532, 2008.

[31] H. Wang, J. Holst, S.-R. Woo et al., "Tonic ubiquitylation controls T-cell receptor:CD3 complex expression during T-cell development," The EMBO Journal, vol. 29, no. 7, pp. 1285-1298, 2010.

[32] S. F. Friend, L. K. Peterson, R. M. Kedl, and L. L. Dragone, "SLAP deficiency increases TCR avidity leading to altered repertoire and negative selection of cognate antigen-specific CD ${ }^{+}$T cells," Immunologic Research, vol. 55, no. 1-3, pp. 116-124, 2013.

[33] L. L. Dragone, M. D. Myers, C. White et al., "Src-like adaptor protein (SLAP) regulates B cell receptor levels in a c-Cbldependent manner," Proceedings of the National Academy of Sciences of the United States of America, vol. 103, no. 48, pp. 18202-18207, 2006.

[34] L. L. Dragone, M. D. Myers, C. White, T. Sosinowski, and A. Weiss, "Src-like adaptor protein regulates B cell development and function," Journal of Immunology, vol. 176, no. 1, pp. 335$345,2006$.

[35] S. J. Holland, X. C. Liao, M. K. Mendenhall et al., "Functional cloning of Src-like adapter protein-2 (SLAP-2), a novel inhibitor of antigen receptor signaling," The Journal of Experimental Medicine, vol. 194, no. 9, pp. 1263-1276, 2001.

[36] G. A. Manes, P. Masendycz, T. Nguyen et al., "A potential role for the Src-like adapter protein SLAP-2 in signaling by the colony stimulating factor-1 receptor," FEBS Journal, vol. 273, no. 8, pp. 1791-1804, 2006.
[37] B. Pakuts, C. Debonneville, L. M. Liontos, M. P. Loreto, and C. J. McGlade, "The Src-like adaptor protein 2 regulates colonystimulating factor-1 receptor signaling and down-regulation," The Journal of Biological Chemistry, vol. 282, no. 25, pp. 1795317963, 2007.

[38] L. M. Liontos, D. Dissanayake, P. S. Ohashi, A. Weiss, L. L. Dragone, and C. J. McGlade, "The Src-like adaptor protein regulates GM-CSFR signaling and monocytic dendritic cell maturation," The Journal of Immunology, vol. 186, no. 4, pp. 1923-1933, 2011.

[39] S.-K. Park, H. Qiao, and M. A. Beaven, "Src-like adaptor protein (SLAP) is upregulated in antigen-stimulated mast cells and acts as a negative regulator," Molecular Immunology, vol. 46, no. 10, pp. 2133-2139, 2009.

[40] S.-K. Park and M. A. Beaven, "Mechanism of upregulation of the inhibitory regulator, src-like adaptor protein (SLAP), by glucocorticoids in mast cells," Molecular Immunology, vol. 46, no. 3, pp. 492-497, 2009.

[41] H.-J. Kim, W. Zou, Y. Ito et al., "Src-like adaptor protein regulates osteoclast generation and survival," Journal of Cellular Biochemistry, vol. 110, no. 1, pp. 201-209, 2010.

[42] S. Sugihara, S. Katsutani, H. Deckmyn, K. Fujimura, and A. Kimura, "Roles of Src-like adaptor protein 2 (SLAP-2) in GPVImediated platelet activation: SLAP-2 and GPVI signaling," Thrombosis Research, vol. 126, no. 4, pp. e276-e285, 2010.

[43] S. Roche, G. Alonso, A. Kazlauskas, V. M. Dixit, S. A. Courtneidge, and A. Pandey, "Src-like adaptor protein (Slap) is a negative regulator of mitogenesis," Current Biology, vol. 8, no. 17, pp. 975978, 1998.

[44] P. Alifragis, Z. Molnár, and J. G. Parnavelas, "Restricted expression of Slap-1 in the rodent cerebral cortex," Gene Expression Patterns, vol. 3, no. 4, pp. 437-440, 2003.

[45] M. A. Takasu, M. B. Dalva, R. E. Zigmond, and M. E. Greenberg, "Modulation of NMDA receptor-dependent calcium influx and gene expression through EphB receptors," Science, vol. 295, no. 5554, pp. 491-495, 2002.

[46] S. Semerdjieva, H. H. Abdul-Razak, S. S. Salim et al., "Activation of EphA receptors mediates the recruitment of the adaptor protein slap, contributing to the downregulation of N-methylD-aspartate receptors," Molecular and Cellular Biology, vol. 33, no. 7, pp. 1442-1455, 2013.

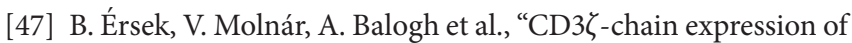
human T lymphocytes is regulated by TNF via Src-like adaptor protein-dependent proteasomal degradation," The Journal of Immunology, vol. 189, no. 4, pp. 1602-1610, 2012.

[48] L. K. Peterson, L. A. Shaw, A. Joetham, S. Sakaguchi, E. W. Gelfand, and L. L. Dragone, "SLAP deficiency enhances number and function of regulatory $\mathrm{T}$ cells preventing chronic autoimmune arthritis in SKG mice," The Journal of Immunology, vol. 186, no. 4, pp. 2273-2281, 2011.

[49] J. U. Kazi, S. Agarwal, J. Sun, E. Bracco, and L. Rönnstrand, “SrcLike Adaptor Protein (SLAP) differentially regulates normal and oncogenic c-Kit signaling," Journal of Cell Science, vol. 127, pp. 653-662, 2014. 


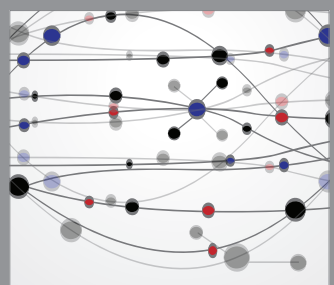

The Scientific World Journal
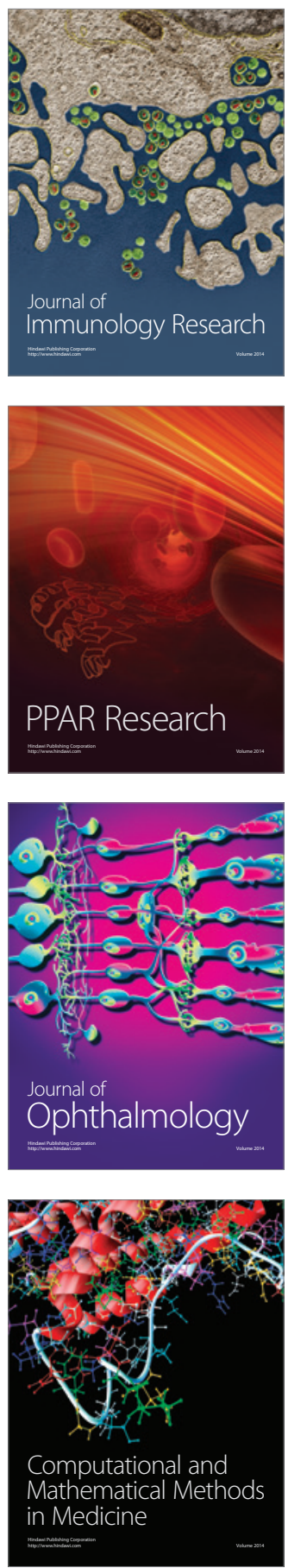

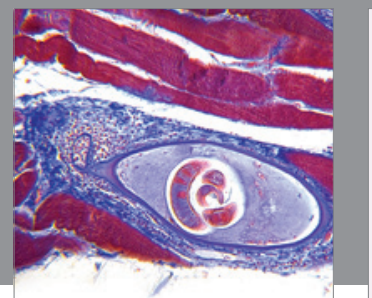

Gastroenterology

Research and Practice
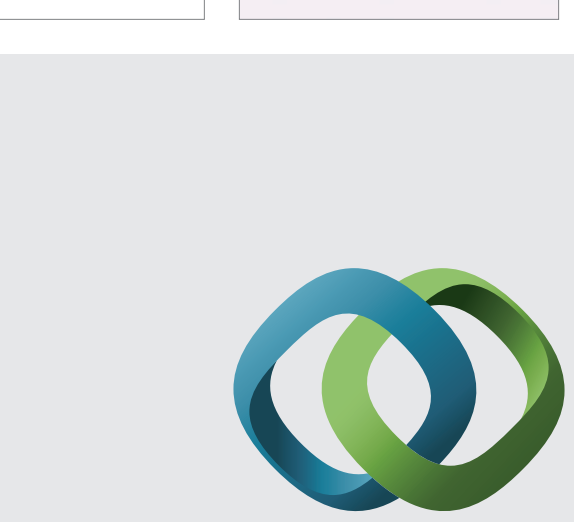

\section{Hindawi}

Submit your manuscripts at

http://www.hindawi.com
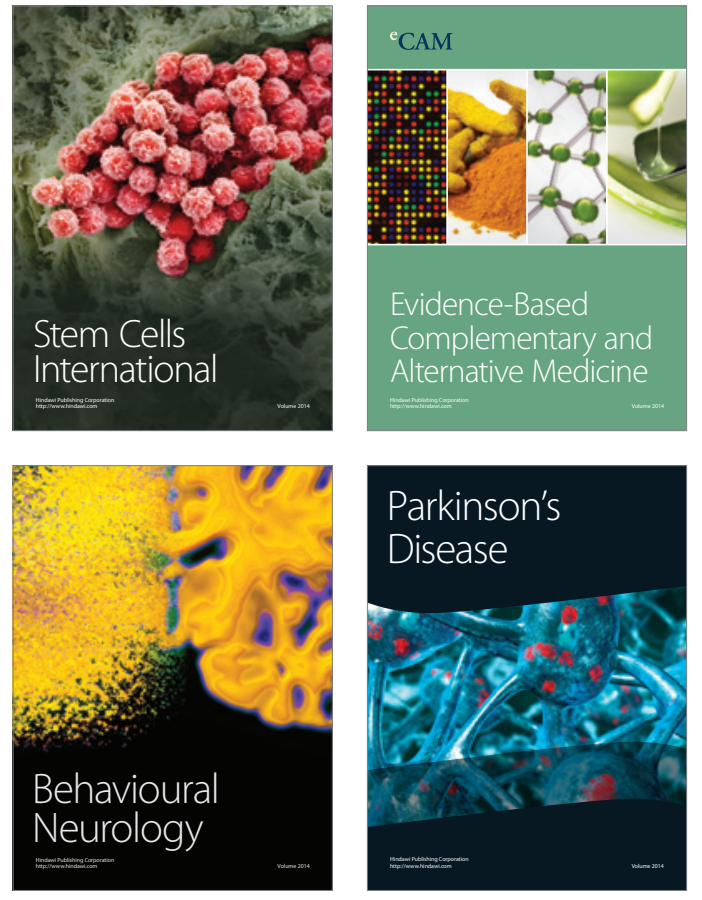
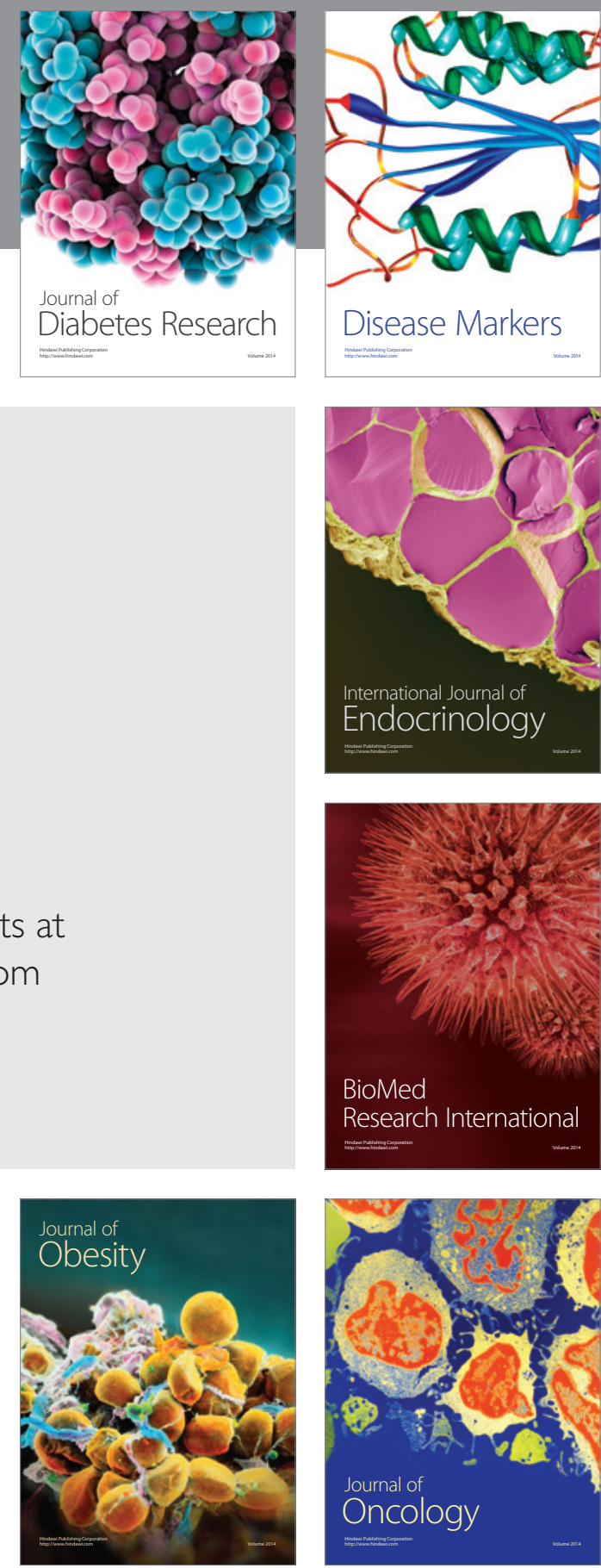

Disease Markers
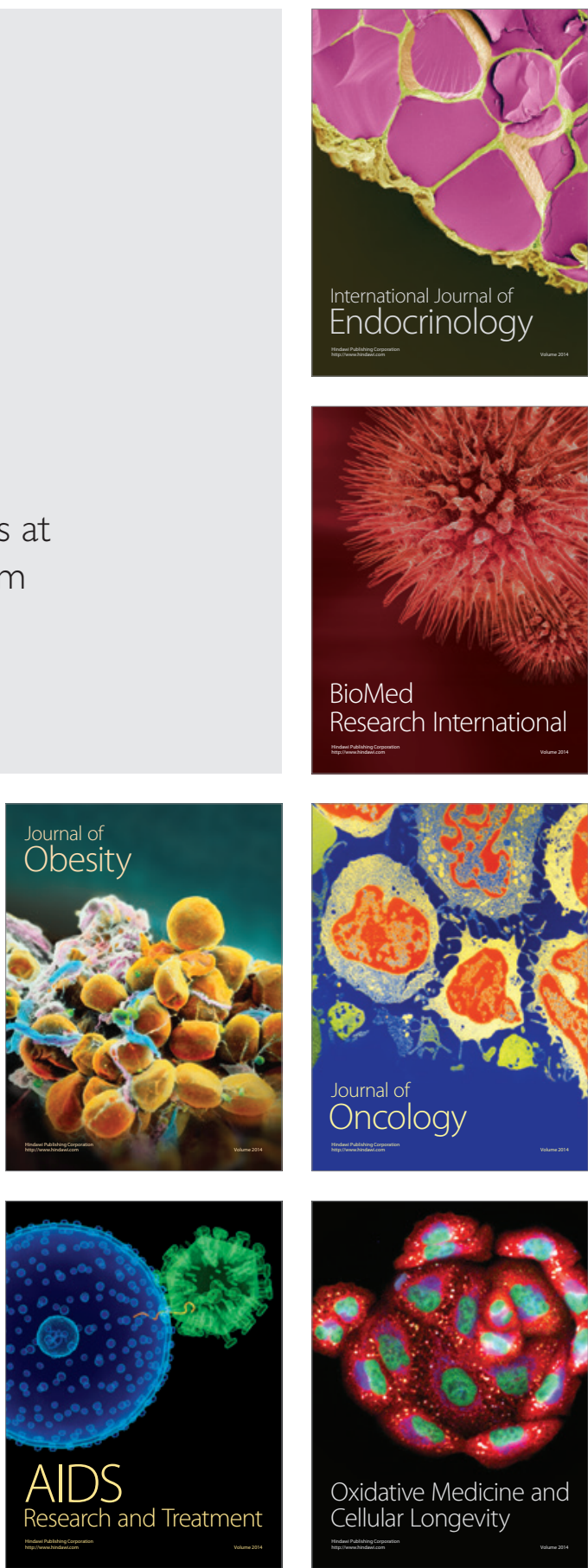